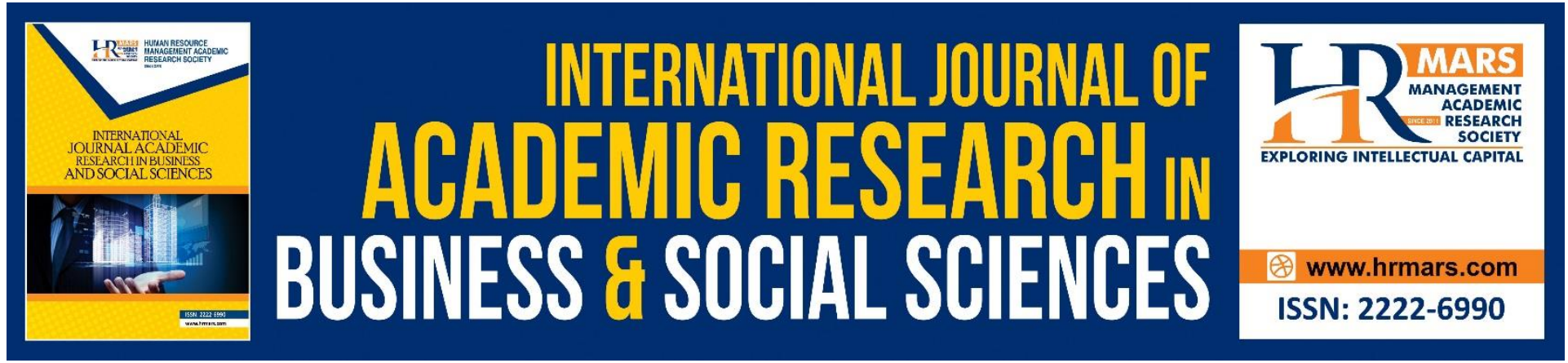

\title{
Escalating the Importance of Preserving the Electronic Records Metadata in Online Business Transaction Activities
}

\section{Ap-azli Bunawan}

To Link this Article: http://dx.doi.org/10.6007/IJARBSS/v11-i8/10505

DOI:10.6007/IJARBSS/v11-i8/10505

Received: 05 June 2021, Revised: 24 June 2021, Accepted: 16 July 2021

Published Online: 06 August 2021

In-Text Citation: (Bunawan, 2021)

To Cite this Article: Bunawan, A. (2021). Escalating the Importance of Preserving the Electronic Records Metadata in Online Business Transaction Activities. International Journal of Academic Research in Business and Social Sciences, 11(8), 78-90.

\section{Copyright: @ 2021 The Author(s)}

Published by Human Resource Management Academic Research Society (www.hrmars.com)

This article is published under the Creative Commons Attribution (CC BY 4.0) license. Anyone may reproduce, distribute, translate and create derivative works of this article (for both commercial and non-commercial purposes), subject to full attribution to the original publication and authors. The full terms of this license may be seen at: http://creativecommons.org/licences/by/4.0/legalcode

Vol. 11, No. 8, 2021, Pg. 78 - 90

Full Terms \& Conditions of access and use can be found at http://hrmars.com/index.php/pages/detail/publication-ethics 


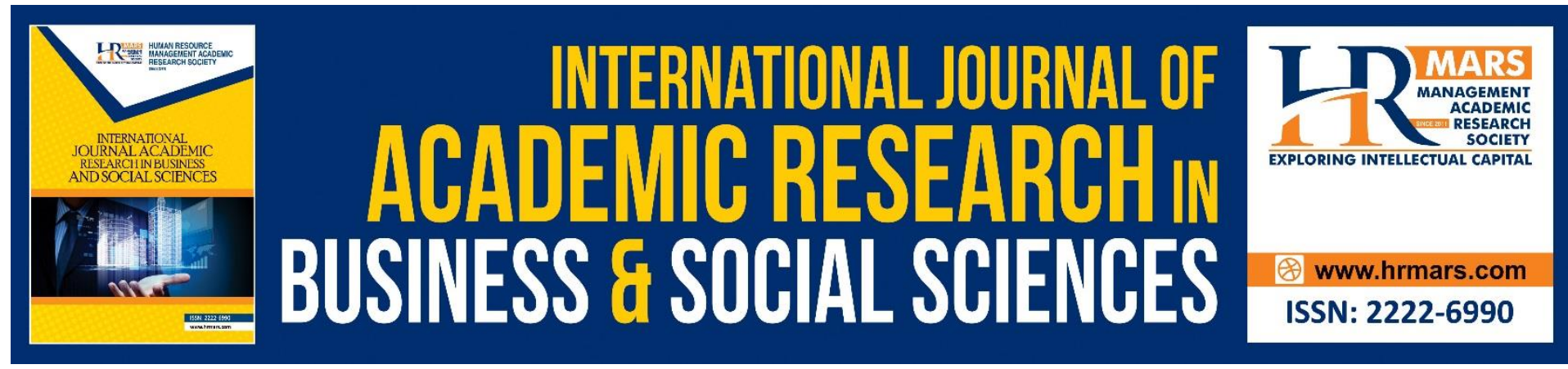

\title{
Escalating the Importance of Preserving the Electronic Records Metadata in Online Business Transaction Activities
}

\author{
Ap-azli Bunawan \\ Faculty of Information Management, Universiti Teknologi MARA (UiTM), Puncak Perdana \\ Campus, Section U10, 40150 Shah Alam, Selangor Darul Ehsan, Malaysia. \\ Email: ap-azli@uitm.edu.my
}

\begin{abstract}
This paper highlighted the importance of preserving the electronic records metadata in online business activities. Due to the COVID-19, all types of businesses are affected and need to change their process of selling the product and services to customers. As the COVID-19 restricted face-to-face transaction or physical attendance activities, the sellers and owners of business choose to conduct their business using online platform. By conducting the business online, the process of providing the evidence of transaction is required. This is where the use of electronic records as evidence of the transaction is applied. In providing the evidence, the preservation of entire metadata of electronic records is also required and becomes the importance aspect in online business. As to identify the importance, the analysis on the literatures is conducted. By using the scoping review technique, the details of the current business process activities and identification of five (5) type of metadata function are presented. The metadata function includes identifying metadata as evidence, asset, tool, application and repository. Last but not least, this paper concludes that the entire metadata function presented has becomes the key aspect in escalating the importance of preserving the electronic records metadata in online business transaction activities.
\end{abstract}

Keyword: Preservation, Electronic Records, Metadata, Online Business, COVID-19

\section{Introduction}

Nowadays, there are many people involved in business activities. These include the business on the aspect of education, banking, food, health and beauty. Most of these activities are conducted by selling either the product or services to other people. In terms of selling activities, the selection of platform used in selling the product and services need to be properly identified. Generally, there are two (2) different types of selling platform that are commonly used in business. The sellers can choose either to sell the product and services using the physical attendance or online selling platform. By choosing the appropriate platform, it can contribute to the successive business outcome and profit.

Recently, sellers have transformed their nature of selling the product and services using the online platform. This transformation not limited to individual seller but it is also applied by other types of organization. Part of the reason is due to the COVID-19 pandemic that suddenly 
attacked the entire world. The COVID-19 pandemic is very critical issues where everyone is ordered to stay at their home as to control the spreading of COVID-19 virus. As everyone stayed at home, the entire business activities that involved physical attendance are ordered to immediate close. This is where many sellers or owner of the business transformed their nature of selling the product and services using the online platform.

As the business is conducted online, there are several aspect need to be managed properly. Part of the aspect is the management of electronic records. In online business, the entire business transaction activities are conducted electronically. This is where the electronic records are used in providing an evidence of the business transaction. These include the electronic records such as purchase order (PO), delivery order (DO), invoice, receipt and payment voucher. These are the types of electronic records that most frequently used in conducting the online business. By properly managed those types of electronic records, the entire details of business transaction can be used as evidence on any legal obligation.

The details of electronic records are presented by the metadata. Each of electronic records contained different types of metadata. There are various types of metadata used in handling the online business transaction activities. Part of the types is administrative, technical, descriptive, use, and preservation metadata. The entire types of metadata are importance to be used in completing the entire business transaction. However, the aspect of preserving the electronic records metadata has becomes a key aspect in formulating the online business transaction evidence. The evidence should contain the overall metadata of electronic records that involved in online business transaction activities. Therefore, preserving the metadata of electronic records is importance and requires special attention.

In order to attain the aspect of preservation metadata, several standard and policy are referred. By referring to these standard and policies, the entire aspect of preservation metadata is analyzed. The analysis is conducted systematically as it has to focus on the importance of preservation metadata. Based on the result of analysis, several importances on preserving the metadata are identified. However, the details of preservation metadata aspect and its importance require proper explanation. This is where the next section is formulated to explain the details of the importance.

\section{Literature Review}

As to explain further on the importance, these are required the details of each domain and concept to be properly discussed.

\section{Concept of Business}

In general, the term Business can be defined as selling a product or services to other people. A business is also can be define as cause; people advocate for the brand, the product or service and a special way of doing business that represents that cause (Rogov, 2020). As stated by Domingo (2018), business is about earning money, giving special attention on marketing could give an organization a favorable profit. Meanwhile, Mitrović et al (2017) stated that modern business is accompanied by many risks and uncertainties, rapid changes in technology and the market, as well as other changes that create a new business ambient in the environment of each business system. This is shows that instead of selling the product, services and gain the profit, business is also facing the risk on the changes of technology that lead to the new environment of business system.

Online Business 
In facing the risk of new environment of business system, the business activities are conducted using online business environment. According to Gutovets \& Pustoshilo (2020), online business is one of the most attractive activities that bring good income. The study conducted by Qamruzzaman (2021) shows that the majority of the entrepreneurs believe that online business is more profitable than a face-to-face business which is certainly true in other digital advanced countries. As stated by Amin (2020), in the time of COVID-19 crisis, online business is not just an option for their customers or business holders but it is the only alternative to do business in this situation. Through these justifications, the online business is proven more profitable as it is also become the main choice of conducting the business during COVID-19 pandemic.

\section{COVID-19 Pandemic}

The impact of COVID-19 has extremely changed many aspect of life including the process in conducting the business activities. Based on Tayade (2021), the COVID-19 has dramatically changed the current business climate and culture where everything becomes volatile and very tuff to survive as earlier. According to Ferdousi (2020), in COVID pandemic world the volatile economy may relies heavily on the online business and services. This is supported by the statement from Tomar \& Chaudhary (2020) where the market share of online business is increasing day by day and the current covid-19 epidemic has given boost to online business. This is shows that the COVID-19 pandemic has triggered the entire businesses to be conducted online. As the most of businesses are conducted online, there is a requirement for providing the evidence on each transaction conducted.

\section{Electronic Records}

In providing the evidence, the electronic records become the main aspect used in ensuring the reliability of the transaction activities. Guto \& Jumba (2021) stated that electronic records as legally verifiable evidence of entitlements, contractual obligations, policies or transactions for the periods required. According to Taiwo (2019) which cited from IRMT (2004), electronic records are the recorded information, documents or data that provide evidence of policies, transactions and activities carried out in an electronic environment. Meanwhile, Beckles (2017) stated that the electronic records are fluid in structure, but they still qualify as records if the associated metadata, or context and content remain unchanged, and the integrity of the systems used to create, distribute, and store them is reliable. Based on the explanations, the electronic records used in online business transaction activities provide reliable evidence as the metadata of electronic records remain unchanged.

\section{Electronic Records Metadata}

As to ensure the metadata is maintained properly, it is important to understand the term of metadata and its relation to electronic records. According to County (2016), metadata is data that provides information about other data. In details, Mazzarese et al (2019) stated that the metadata is a set of data that describes and gives information about other data, including raw data, sample data, or analyst data. In term of its relation, Chiwanza \& Mutongi (2017) stated that it is importance to identify the metadata that needs to be captured and maintained with electronic records of enduring value if they are to remain identifiable and accessible over time. Sequentially, Florida (2021) stated that metadata in the context of records management is data describing context, content, and structure of records and their management through time, and performs other administrative tasks easily and in compliance with all legal 
requirements. This is where metadata is preserved in the data layer, supporting the error and log strategies to handle errors and pattern events (Oliveira \& Belo, 2017). Based on the discussion, the preservation of metadata becomes an importance aspect in providing the evidence of business transaction activities.

\section{Preservation of Metadata}

In terms of preservation of metadata, there are many perspectives in understanding the terms are highlighted by scholars. From the perspectives of Web Content Archiving and Storage, Mulauzi (2019) stated that the key recordkeeping requirements include collecting website metadata, preserving content in original file formats, and retaining materials for records requests and retention. In term of policy, Lemieux (2020) stated that disseminate and implement an information governance policy to mandate the creation, management and preservation of electronic records and associated metadata across the government or the organisation. Meanwhile, according to Lavoie (2019), the Open Archival Information System (OAIS) information model is built around the concept of an information package, which consists of the object that is the focus of preservation, along with metadata necessary to support its long-term preservation, access, and understandability. As stated by Giaretta (2020), active data management plans determined the metadata is needed for preservation in any given situation and the important to capture the metadata as soon as the data are created rather than waiting to collect them at the end or attempting to collect them later. Through these justifications, several justifications on the importance of preserving the metadata are presented. However, the justifications are doubtful and require other details or clarification to be conducted.

\section{Metadata as Evidence}

The metadata can act as evidence as long as the entire metadata used in handling the online business transaction activities are properly preserved. According to Krishnan \& Shashidhar (2021), in term of digital forensic, internet browsers that render websites and WebPages are of interest to forensic investigators as browser metadata can be used extensively as forensic evidence in all kinds of cases. As stated by Ansari (2018), metadata defines the evidence for a particular rule implementation and each set of methods are associated some kinds of rules. Meanwhile, as per Tilton et al (2020), metadata brings context to the analysis, but the form of the metadata will define which kinds of analysis can use metadata as context and, therefore, evidence. Based on the discussion, metadata is proven can be used as evidence in various types of activities.

\section{Metadata as Asset}

Instead of becoming the evidence, the metadata is also used as an asset of business activities. As stated by Hardjono et al (2020), the asset profile metadata is used when a regulated entity seeks to issue digital or virtual assets in a jurisdiction that recognizes the asset type. This followed by Horsch (2021) that presented the sample cases of data producer and data consumer communication which proven as an examples on the importance of metadata as semantic asset, and therefore as a mode of fixed and negotiated communication. However, Radini et al (2018) stated that the complexity of the metadata asset referring to structural metadata asset or intensional data representation and in terms of hugeness and lack of a direct control due to several sources are administrative come with their own semantics. Meanwhile, Nicolescu (2019) explained the value that metadata, or little data, brings to big 
data is in the structure and it meaning provides by serving as asset discovery by identifying data and allowing them to be found by relevant criteria. Through these justifications, the metadata is highly valuable and proven as important asset to be properly preserved.

\section{Metadata as Tool}

Identifying the metadata as tool, Mahony \& Wenning (2020), stated that metadata is one tool used in addressing the issues of data which also allows data owners to maintain control of their data. Subsequently, according to Azhar (2021), a file-based format is recommended for this metadata tracking function where the archive metadata field is an essential tool for producers or vendors data entry and retrieval function. As stated by Patzák et al (2018), some metadata have to be defined by user or simulation tool and some can be automatically collected by the platform. Currently, Crusoe et al (2021) presenting the Common Workflow Language (CWL) description, the metadata about the tool description of authors encourages attribution of their efforts. As being discussed, the use of metadata as a tool in completing many types of business activities is justified. However, instead of tool, the online business transaction activities also require an application as the platform for conducting the online transaction activities. Therefore, the details on the usage of metadata as application need to be discussed adequately.

\section{Metadata as Application}

As metadata identified as application, Fleischer \& Combelles (2019) are presented the realization of metadata concepts through ontologies in a business application. According to Satija et al (2020), the ideation of application-specific metadata application profiles (APs) is based on the principles of functionality, modularity and agile data management. On top of that, by Ravi (2017) stated that the metadata application should be a major component of the digital information processing. Subsequently, Chunqiu (2018) defined the metadata schema as a semi-formal description scheme that defines syntactic, structural, and semantic features of metadata used for an application. Based on the discussions, the overall aspect of metadata as application is properly presented. However, the process in storing the entire metadata in a dedicated storage produced by applications still unclear. This is where the aspect of storing a metadata in a dedicated storage or repository requires detail explanations.

\section{Metadata as Repository}

As required, there is several aspect of metadata as repository presented by scholars. According to Demidova et al (2018), the metadata repository is responsible for collecting data, storing data, and presenting data for analysis. As per Tekwani et al (2017), the metadata repository contains the data about the data description of the source data, the target data, and how the source data has been converted to the target data. Sequentially, Man et al (2019) stated that metadata repository is a central storage area for multiple types of semi-structured data integration architecture which provides information about data sources for users. As for the institutional metadata repository, Smale et al (2020) stated that it can enable the creation of prepublication records, application of an appropriate license, reservation of a DOI (Digital Object Identifier) and a data citation. Through the discussion, the entire aspects of metadata as repository are appropriately explained.

Overall, there are five (5) importance of metadata are identified namely evidence, asset, tool, application and repository. The identification has justified that the function of metadata and its relation to any related activities are properly applied and executed. Based on this 
execution, it is proven that the importance of preserving the metadata of electronic records is adequately justified. However, in completing the overall process of explaining the importance and preservation aspect of electronic records metadata, the section of Result is formulated. Through the section, the details of each importance of metadata are completely presented and explained.

\section{Methodology}

In analyzing and exploring on the specific field of study, there are many technique are applied. Parts of the technique that are commonly applied by scholars are systematic, meta-analysis and scoping review technique (Fahim et al., 2018; Neubauer et al., 2019: Chen \& Konkle, 2020). According to Van Dalen (2021), a suboptimal literature search can compromise the validity of the conclusions of the systematic review as it can introduce reporting bias. In terms of meta-analysis, Sutrisno \& Dularif (2020) defined the meta-analysis as a statistical method to integrate results from different studies and identify the relationships appearing in the previous studies on the same topic. As for the scoping review, Xinyan et al (2020) stated that a scoping review is an effective method of evidence identification and synthesis, which can provide a basis for the further development of a certain field. Through these explanations, the scoping review technique are selected and applied instead of the systematic and metaanalysis. As becoming the effective method suggested by scholars, the scoping review technique also provides other valuable impact and advantages to the researchers.

In details, a scoping review is a rapid gathering of literature in a given subject area where the aims are to accumulate as much evidence as possible and map the results (Pottie et al., 2018). According to Guden \& Bellen (2020), the scoping review is an effective means of highlighting the relevant literature to the researcher, with the aim of rapid mapping of key concepts underpinning a research area and a robust method of identifying primary and secondary sources of literature. Significantly, as stated by Roomaney et al (2020), the scoping review is the most appropriate method to conduct this comprehensive exploration of the current evidence which may be sparse due to the rarity of the condition. Based on the discussions, the selection of scoping review technique in this paper is firmly justified.

\section{Result}

In presenting the overall finding gathered from literatures, this section is designed. The findings include presenting the justification on the importance of preserving the metadata and the relation of each subject domain presented in this paper. In ensuring the entire findings are adequately presented, the figure on the overall of findings is illustrated. 


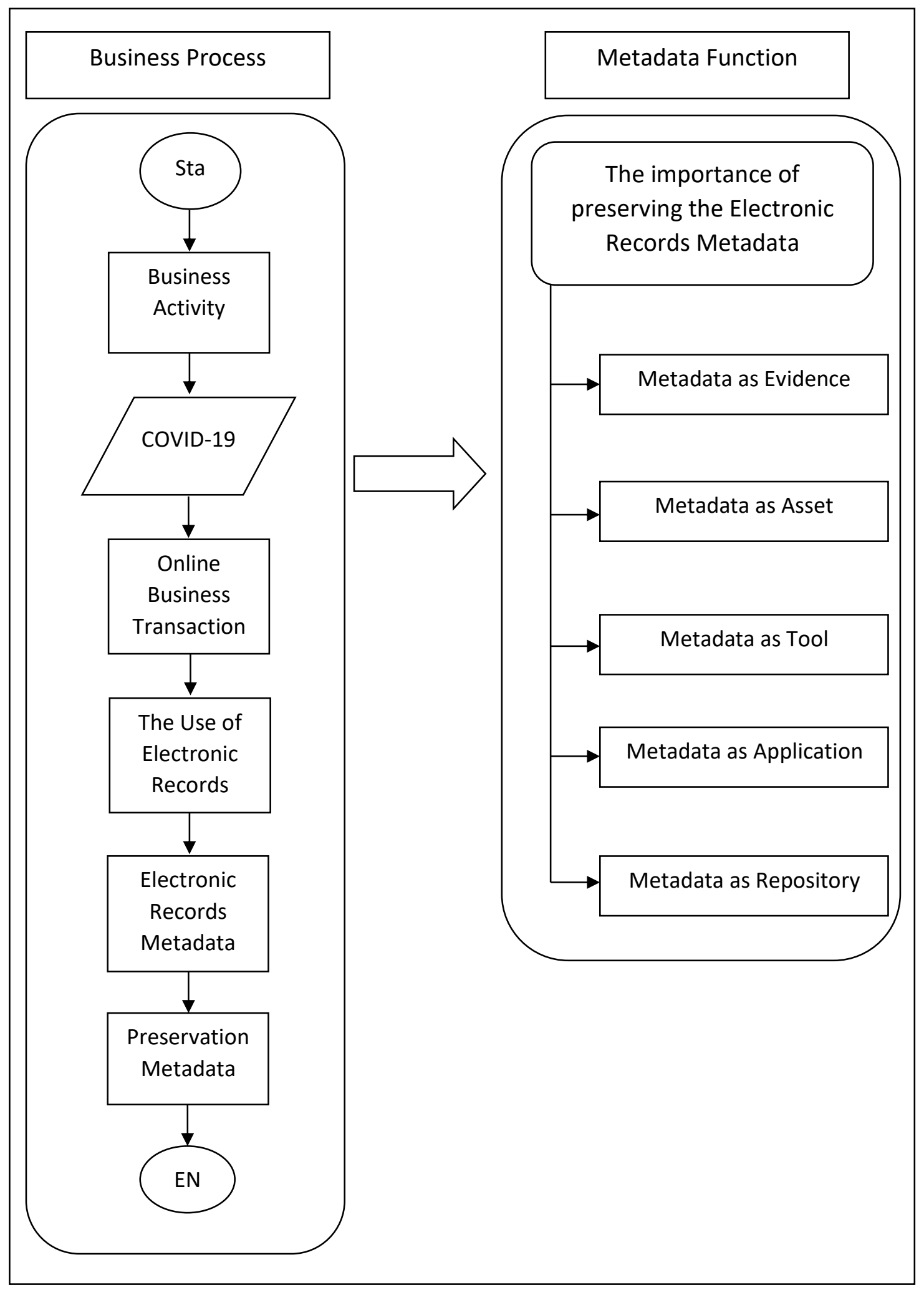

Figure 1: The importance of Preserving the Electronic Records Metadata

Based on the figure 1, the importance of preserving the electronic records metadata that triggered on the latest trend of business conducted is completely presented. The figure begins with presenting the flow of the "Business Process". By presenting the "Business Activity" as the core ideas of the business process flow, this is suddenly changed due to the "COVID-19" 
pandemic attack. As the pandemic attack, the business activities are changed to be conducted using the "Online Business Transaction" approached. This is then involved "The Use of Electronic Records" as a proof of online business transaction conducted. In ensuring the electronic records can act as a proof of the transaction, the "Electronic Records Metadata" must be preserved. This is where the aspect of "Preservation Metadata" is highlighted as the last activities of the business process flow. However, the importance of preserving the metadata in online business transaction activities is still unclear. Therefore, the details on the importance of preserving the metadata are also presented in the above figure as "Metadata Function"

In presenting the function of metadata, there are five (5) functions are indentified. There are "Metadata as Evidence", "Metadata as Asset", "Metadata as Tool", "Metadata as Application" and "Metadata as Repository". In each of the function, there are aspects that need to be focused. This includes applying the aspect of metadata execution and operation in different types of online business activities. As applied, the function of metadata still remains valuable to be used as a proof of the entire business transaction activities. Through these justifications, this is proven that the process in preserving the metadata of electronic records in online business transaction activities is importance and contributes greatest significance.

\section{Conclusion}

In business, the process in ensuring the business can gain a profit and valuable for the society are based on the nature of business applied. The selections of platform in conducting the business also reflect the profit and valuable output produced. In focusing on the single platform of businesses, the entire aspect of business objectives could be difficult to be achieved. This is due to the latest style of living where the face-to-face business transactions are restricted. As restricted based on the COVID-19 pandemic attacked, the sellers and business owners do not have a choice in conducting their business. This is where the online business should be the priorities.

In conducting the online business, there are many aspects require a special attention. This means that the entire online business conducted should focus on producing the proof of transaction online. As to provide the proof online, this is where the use of electronic records is required. The electronic records become the main focus where each of the bank transfer records, order records, delivery records or any type of records used are becoming the main proof of the business transaction. This is also includes preserving the entire of metadata of electronic records to provide an authentic evidence of the transaction. This is part of metadata function that nurtures the understanding on the importance of preserving the metadata in online business transaction activities.

Overall, there are five (5) metadata functions that importance for online business. These include metadata as evidence, asset, tool, application and repository. By having proper understanding on each function of metadata, the process in providing the authentic evidence and reliable records would adequately achieved. Last but not least, achieving the authentic evidence of business transaction activities would not be achieved without nurturing proper understanding on the importance of preserving the electronic records metadata.

The COVID-19 attacked has triggered the sellers and business owners urged immediate action in changing their current nature of selling the product and services to online business platform. As an impact of this immediate action, the issues of providing the evidence of online business transactions activities using the electronic records are occurred. Through the occurrences of the issues, this paper is designed to identify the possible solution that could 
be used in solving the issues driven. In identifying the solution, there are five (5) types of metadata of electronic records are identified as an importance aspect in providing the evidence of online business transaction activities. This has led to the illustration of figure which contained the details explanation on the relation of online business activities which has reflected the requirement of preserving the electronic records metadata. By applying the basic concept of business and part of essential theories in records and metadata, this figure can be applied genuinely by any sellers and business owners specifically in conducting their business online. This is also could becomes part of the business reference model for sellers and business owners in providing the reliable evidence while handling their online business transaction activities. Overall, the entire findings presented in the figure could generate an added value to the body of knowledge regarding the relationship between the concept of online business and the importance of preserving the electronic records metadata.

\section{References}

Amin, A. (2020). Youth entrepreneurship and online business: a study on economic effects of coronavirus outbreak (COVID-19) in Dhaka City. International Journal of Career Development, 3(2020), 31-46.

Ansari, A. (2018). Case-study soa design and development for bepet. International Journal of Novel Research and Development (IJNRD), 3(12), 37-44.

Azhar, R. R. B. R. (2021). The impact of industrial revolution 4.0 on television content production and broadcasting in malaysia: challenges and opportunities. BERJAYA Journal of Services \& Management, 15, January 2021, 25-36.

Beckles, C. A. (2017). Protecting the once-'silent' record. Information Management (C2017 ARMA international. http://www.arma.org.

Chen, S. M., \& Konkle, A. (2020). Scoping review of the effects of dietary supplements on postpartum depression. Journal of Health and Medical Sciences, 3(2).

Chiwanza, K., \& Mutongi, C. (2017). Strategies for managing electronic records in ODL. EPRA International Journal of Research \& Development (IJRD), 2(7), 28-37.

Chunqiu, L. (2018). A study on provenance description of metadata schemas for longevity of metadata in networked information environment. https://core.ac.uk/download/pdf/159404934.pdf

County, J. (2016). Administrative policies \& procedures regarding public records request. http://www.co.josephine.or.us/Files/10-25-16\%20General\%20Minutes.pdf

Crusoe, M. R., Abeln, S., losup, A., Amstutz, P., Chilton, J., Tijani, N., Ménager, H., SoilandReyes, S., \& Goble, C. (2021). Methods included: standardizing computational reuse and portability with the common workflow language.

https://research.vu.nl/ws/portalfiles/portal/130792587/2105.07028v1.pdf

Demidova, A. V., Kuznetsov, Y. A., \& Fomin, M. B. (2018). Designing Multidimensional Information Systems Using the Data Vault Methodology. In CEUR Workshop Proceedings (pp. 33-39).

Domingo, A. V. (2018). Product, pricing and promotional strategies of restaurants in Nueva Ecija: An Assessment. International Journal of Advanced Engineering, Management and Science, 4(11), 268-271.

Fahim, C., Wagner, N., Nousiainen, M. T., \& Sonnadara, R. (2018). Assessment of technical skills competence in the operating room: a systematic and scoping review. Academic Medicine, 93(5), 794-808. 
Ferdousi, B. (2020). Data security concerns and consumers' trust in online business. Proceedings of the 5th NA International Conference on Industrial Engineering and Operations Management Detroit, Michigan, USA, August 10 - 14, 2020. http://www.ieomsociety.org/detroit2020/papers/480.pdf

Fleischer, A., \& Combelles, J. (2019). The evolution of metadata concepts from metadata harvesting to ontology modeling. ACM Chapter Proceedings on Educational Technology, Language and Technical Communication Theme: Information Design and Management, 18-23.

Florida, A. (2021). Managing Florida's Public Records. State of Florida. https://files.floridados.gov/media/704224/managing-floridas-public-records.pdf

Giaretta, D. (2020). Preserving and using digitally encoded information as a foundation for achieving the Sustainable Development Goals. University of London Press, Institute of Commonwealth Studies. https://www.jstor.org/stable/pdf/j.ctv14t45v5.15.pdf

Guden, J., \& Bellen, J. (2020). A scoping review of the peer-led team learning to learnerparticipants and peer leaders in STEM courses. International Journal of Research Studies in Education, 9(5), 1-13.

Guto, R., \& Jumba, A. H. (2021). Relationship between electronic records management and public organization credibility: critical analysis of literature review. Journal of African Interdisciplinary Studies, 5(3), 52-67.

Gutovets, A., \& Pustoshilo, Y. (2020). Internet as a platform for sales. http://edoc.bseu.by:8080/bitstream/edoc/85357/1/Gutovets_103_104.pdf

Hardjono, T., Lipton, A., \& Pentland, A. (2020). A contract service provider model for virtual assets. in 6th international workshop on $p 2 p$ financial systems, London. https://hardjono.mit.edu/sites/default/files/documents/hardjono-p2pfisy-2020.pdf

Horsch, M. (2021). Research data infrastructures and engineering metadata. https://link.springer.com/content/pdf/10.1007/978-3-030-68597-3_2.pdf

International Records Management Trust (IRMT) (2004). The e-records readiness tool. https://www.irmt.org

Krishnan, S., \& Shashidhar, N. (2021). Interplay of Digital Forensics in eDiscovery. International Journal of Computer Science and Security (IJCSS), 15(2), 19.

Lavoie, B. (2019). The Open Archival Information System (OAIS) Reference Model: Introductory Guide (2nd Edition). The Digital Preservation Coalition, Great Britain.

Lemieux, V. (2020). Transparency in the 21st century: the role of records in achieving public access to information, protecting fundamental freedoms and monitoring sustainable development. University of London Press, Institute of Commonwealth Studies.

Mahony, A. O., \& Wenning, R. (2020). Multi-owner data sharing for analytics and integration respecting confidentiality and owner control. https://mosaicrown.eu/wp-content/uploads/2020/09/D3.1.pdf

Man, M., Sabri, I. A. A., Jalil, A., Ali, N., \& Muhamad, S. (2019). Information integration architecture system for empowering rural woman in Setiu Wetlands, Terengganu, Malaysia. Journal of Sustainability Science and Management, 14(1), 77-86.

Mazzarese, R. P., Bird, S. M., Zipfell, P. J., \& Dong_, M. W. (2019). Chromatography data systems: perspectives, principles, and trends. LC GC North America, 37(12), 852.

Mitrović, S., Milisavljević, S., Brkljač, N., Vrhovac, V., \& Žižakov, M. (2017). The importance of an adequate organization management system in contemporary business conditions. https://www.iim.ftn.uns.ac.rs/is17/papers/56.pdf 
Mulauzi, F. (2019). Application of Information and Communication Technologies (ICTs) in Records Management. Library and Information Association of Zambia Journal (LIAZJ), $5(1 \& 2), 41-54$.

Neubauer, N. A., Laquian, K., Conway, C., \& Liu, L. (2019). What do we know about best police practices for lost persons with dementia? A scoping review. Neurodegenerative Disease Management, 9(6), 319-330.

Nicolescu, I. A. (2019). From big data to data: the value behind metadata governance in statistics. Economic Convergence in European Union, 309.

Oliveira, B., \& Belo, O. (2017). Validating ETL patterns feasability using alloy. In DATA (pp.200207).

https://pdfs.semanticscholar.org/9ae8/f1f91238fa1ae32f9521d8a3fab8f41f5c65.pdf

Patzák, B., Milauer, V., \& Horák, M. (2018). MuPIF: Multi-Physics Integration Platform. In Proceedings of the ECCM.

http://congress.cimne.com/eccm_ecfd2018/admin/files/filePaper/p782.pdf

Pottie, K., Ratnayake, A., Alghazali, I., Ahmed, R., \& Veronis, L. Social media networking and information behaviours in youth refugees: A protocol for.

https://methods.cochrane.org/equity/sites/methods.cochrane.org.equity/files/public/ upload s/social_media_protocol_jan_4_2018.pdf

Qamruzzaman, M. (2021). Online business growth in Bangladesh during COVID-19: Owner perception assessment. International Journal of Multidisciplinary Research and Growth Evaluation, 2 (2), 217-224.

Radini, R., Scannapieco, M., \& Tosco, L. (2018). The Italian integrated system of statistical registers: design and implementation of an ontology-based data integration architecture. https://www.istat.it/it/files/2018/11/Scannapieco_original-paper.pdf

Ravi, K. B. (2017). Digital libraries future and development issues. International Journal of Engineering and Management Research (IJEMR), 7 (12).

Rogov, V. (2020). Emotions in business. http://edoc.bseu.by:8080/bitstream/edoc/85388/1/Rogova_123_125.pdf

Roomaney, I. A., Kabbashi, S., \& Chetty, M. (2020). Enamel renal syndrome: a scoping review protocol. https://doi.org/10.21203/rs.3.rs-93115/v1

Satija, M. P., Bagchi, M., \& Martínez-Ávila, D. (2020). Metadata management and application. Library Herald, 58(4), 84-107.

Smale, N., Unsworth, K., Denyer, G., Magatova, E., \& Barr, D. (2020). IJDC General Article. International Journal of Digital Curation, 15(1), 30.

Sutrisno, T., \& Dularif, M. (2020). National culture as a moderator between social norms, religiosity, and tax evasion: Meta-analysis study. Cogent Business \& Management, 7(1), 1-20.

Taiwo, B. I. (2019). Electronic records management implementation: factors affecting organizational readiness. In Information and Knowledge Management, 9(6), 1-14.

Tayade, P. N. (2021). Covid-19 and its impact on e-commerce in India-a critical study. https://eprajournals.com/

Tekwani, D., Agrawal, S., \& Deshmukh, R. K. (2017). An Insight on Data Warehousing for Product Marketing. Indian Journal of Scientific Research, 14(2), 464-466.

Tilton, L., Alexander, E., Malcynsky, L., \& Zhou, H. (2020). the role of metadata in American studies. Polish Journal for American Studies, 14, 149-272. 
Tomar, S. S., \& Chaudhary, S. (2020). A study on factors affecting the online shopping of gardening products. International Journal of Multidisciplinary and Current Educational Research (IJMCER), 2(6), 25-29.

Van Dalen, E. C. (2021). Handbook for systematic review development. Version 1.

Vijaykumar, K. A. (2018). A study on cyber crime and its investigation with special reference to pune district.

https://shodhgangotri.inflibnet.ac.in/bitstream/123456789/6570/1/01_synopsis.pdf

Xinyan, W., Yinuo, W., Hanyi, Y., \& Aslam, M. S. (2020). The scoping review of Chinese and western medicine treatment of diabetic foot in Asia. Universal Journal of Pharmaceutical Research (UJPR), 5(2), 47-53. 